\title{
UN NOUVEAU DISPOSITIF POUR MENSURATION DANS LES TRAVAUX BIOMETRIQUES
}

\author{
W. Besnard
}

Le problème de la mensuration en biométrie a toujours été un point délicat à cause du manque d'un appareil pratique et surtout simple, qui en même temps garantirait une certaine précision. Il semblerait que prendre plusieures mesures, disons, d'un poisson, n'est pas sorcier, ce qui d'ailleurs est parfaitement vrai quand on a tout le temps pour le faire. Mais quand il s'agit de centaines de spécimens par série et il s'agit d'une quinzaine de mesures pour chacun et le temps est chaud, la situation change sérieusement.

D'autre part, si la mesure de la longueur totale ou de la hauteur est relativement simple, les autres deviennent suivant leur direction, position ou étendue de plus en plus difficiles et dans certains cas incertaines.

Par exemple, si le diamètre de l'oeuil est assez simple à prendre, la distance préorbitale ou prédorsale ne le sont pas du tout, vu qu'ici intervient la courbure du corps. Il semblerait que dans ces cas on devrait mesurer la longueur de la ligne reliant les deux points en suivant la surface du corps, ce qui est pratiquement impossible, à moins de se servir d'un eurvimètre. Prendre ces mesures à la pointe sèche amène à des erreurs individuelles et instrumentales difficiles à évaluer et souvent considérables. Ainsi, la longueur des branches du compas, leur écartement, l'épaisseur du spécimen, ete., sont des causes déjà importantes d'erreurs. Par exemple la distance préorbitale d'un Mugilidé ne peut être faite à la pointe sèche qu'avec une approximation à l'estime, cas ou l'erreur individuelle peut s'avérer sérieuse.

Ces difficultés et leur importance dans les recherehes biométriques apparaissent déjà dans la serie de très intéresssants travaux de F. P. Navarro sur la Sardinella aurita des Baléares et des Canaries. En effet, au début il a usé des techniques habituelles, en se servant surtout de la pointe sèche. Plus tard ayant constaté que la méthode semblait être defectueuse il a introduit la mesure en projection verticale. Il l'obtenait en utilisant le tour de main suivant: "...un cadre en celludoïd sur les bords d'une euvette, que l'on déplace; un guide glisse le long du bord inférieur de la euvette. Les autres valeurs sont prises directement à la position de la $\mathrm{D}$ et $\mathrm{V}$, les résultats obtenus par ces deux procédés ne sont pas absolument comparables..." (Nuevos estudios sobre la Alacha (Sardinella aurita Cuv., \& Val.,) de Baleares y de Canarias. Por Francisco 
de P. Navarro. Instituto Español de Oceanografia. Notas y Resúmenes; Série II, n. ${ }^{\circ}$ 60. Junio de 1932, Madrid, pp. 5 \& 6). De notre part nous avons utilisé un dispositif plus ou moins semblable à celui de la cuvette, mais nous avons rencontré non seulement l'inconvénient que signale Navarro, mas encore un autre plus important - la nécessité que la mesure et la lecture soient executées en position strictement verticale. En effet, le moindre écart de cette position, ce qui au cours de la mensuration est psychologiquement très facile, amène des erreurs d'autant plus considerables que la distance entre l'object et le cadre est plus grande. D'autre part, l'utilisation au cours de la mensuration d'un seul spécimen de trois méthodes differentes détermine une perte de temps précieux. Toujours à la recherche d'un appareil permettant toutes les mesures, et qui soit simple, peu encombrant, facile à construire et surtout à manier sans fatigue pour l'opérateur, nous a amené à établir plusieurs prototypes d'appareils de mesurer, basés surtout sur des eadres glissants (2 eadres et un curseur) avec répérage optique au moyen d'un tube muni de 2 croix servant d'alidade verticale. Malheureusement tous ces appareils étaient rélativement encombrants et pour donner une suffisante précision éxigeaient une construction et un ajustage parfaits. Enfin, ce qui est très important, e'est que le pointage à travers le tube optique est fatigant et après la mensuration de plusieures dizaines de spécimens, la superposition des deux croix et du point à observer deviennent difficiles et des erreurs s'en suivent.

Enfin, tout récemment, nous sommes arrivés à la concéption d'une règle qui nous semble répondre à toutes les éxigeances de la mensuration orthogonale, et qui est d'une simplieité telle qu'il semblerait inutile de la décrire. Mais comme nous savons par experience comme il a été difficile d'arriver à ce résultat quasi enfantin, nous nous permettons de la décrire même avant la construction du modèle définitif en métal. Il se peut que par cela nous éviterons à quelques chercheurs, qui pourraient s'intéresser à ce dispositif, une perte de temps et de la fatigue inutiles.

Comme on le voit sur la photographie (phot. 1), l'appareil consiste en une règle soutenue par quatres pieds à joints ajustables, ce qui permet de modifier son hauteur suivant le volume et l'épaisseur du spécimen à mesurer.

La règle ne porte aucune division, ne servant que de guide et support pour deux curseurs pouvant se déplacer sur toute sa longueur. Ces deux curseurs qui forment la partie essentielle de l'appareil, sont differents. Les deux portent des stylets pointeurs, mais ce n'est que l'un d'eux qui sert à la lecture.

Le curseur de gauche (pour le prototype présent) qui au cours de la mensuration n'est déplacé que quand on change le point initial de la mesure, p. ex quand on a terminé les mesures partant du rostre, peut être fixé par une vis de serrage (fig. 1 \& 2 , f) consiste en un cavalier portant un stylet de pointage (fig. 1 \& 2, a) retenu par son guide, une boîte contenant un mètre métallique souple (fig. $1 \& 2$, e, c) et une languette métallique servant d'indicateur de lecture (fig. 1 \& 2, d). 
Le eurseur de droite se résume à un eavalier, au second stylet de pointage et sert de point de fixation de l'extremité zéro du ruban du mètre.

Les stylets pointeurs (fig. 1 \& 2 , a) sont des tiges de section réctangulaire, plate ou ovale, mais non cylindrique, pour assurer la direction de la pointe. Son bout inférieur est recourbé et effilé (fig. $1 \& 2$ ) quant à l'extremité supérieure, elle porte un bouton pour faciliter la pression du doigt. Le guide retient le stylet en deux points distants, interdisant toute déviation, mais assurant les mouvements verticaux sans aucun frottement. Entre les deux points de guidage, sur le corps du stylet est enfilé un ressort à boudin, arrêté par une elavette et qui assure le retour du stylet, après pointage, à sa position de repos. Le bout des stylets sont recourbés inversement pour faciliter les mensurations des petites distances et aussi pour simplifier la construction de l'appareil et son réglage à 0 comme on le verra plus bas.

Le corps du curseur de gauche. A' côté et à l'arrière du stylet pointeur et exactement au dessus de la règle support, se trouve une boîte, où est logé un mètre métallique de poche (fig. 1). Il est essentiel que le ruban de ce mètre soit recouvert de vernis élastique blane, qui tout en protégeant le ruban de la rouille facilite grandement la lecture. Le côté droit de la boîte, c.a.d. celui qui se trouve à côté du stylet pointeur et à l'aplomb de la règle, porte une fente laissant passer le ruban (fig. 1 $\& 2, \mathrm{c})$. Le côté opposé au stylet et son guide sont amovibles et retenus par des vis, pour permettre de graisser ou changer le mètre quand cela est nécessaire. Au dessus de la fente est fixée une lame métallique (fig. 1 \& 2, d) servant d'indicateur de lecture. Un point important: le bout de l'indicateur de lecture doit se trouver exactement à la verticale de la pointe du stylet pointeur.

Enfin, les deux côtés de la boîte du mètre se prolongent vers le bas, en formant les deux guides du curseur, enfermant la règle. Ils sont reliés en bas par une pièce portant la vis de fixation.

Curseur de droite: Ce dernier n'est composé, comme nous l'avons déjà dit plus haut, que du cavalier eurseur portant son stylet pointeur à bout inversé par rapport à celui de gauche, et à l'avant, le point de fixation du bout zéro du ruban du mètre. La vis de fixation ici n'est pas essentielle si l'on travaille de gauche à droite. L'essentiel est que le zéro du ruban se trouve exactement à la verticale du bout pointeur du stylet.

Réglage du dispositif. Les pièces montées et placées sur la règle support, si les verticales mentionnées plus haut sont respectées, doivent être automatiquement reglées, c.a.d. qu'en rapprochant les curseurs, au moment où le bout des stylets pointeurs viennent à se toucher, l'indicateur de lecture doit être exactement à zéro. Si ce résultat n'est pas obtenu, il n'y a qu'à procéder à cet ajustage. On conçoit bien, que quand l'appareil est fabriqué en métal, et surtout si la règle support est profilée en section triangulaire, l'appareil se règle une fois pour toutes et bien 
entretenu peut donner des lectures à moins d'un $1 / 2$ millimètre, précision exagerée en biométrie, mais qui peut être utile dans des mensurations d'alevins.

Le dispositif est tellement simple qu'il est inutile de donner son mode d'emploi. Mais en tout eas on peut attirer l'attention sur deux tours de main utiles. Premièrement on place le spécimen où bien dans une cuvette émaillée où, ce qui est mieux, sur une plaque de verre blane. Le prémier mouvement est de régler la position du spécimen dans la direction de la mesure à effectuer, en déplaçant la plaque de manière que la ligne à mesurer tombe d'aplomb et soit paralèlle au bord de la règle support. Puis, en manipulant le stylet pointeur du curseur gauche, la vis de fixation desserrée, on amène la pointe du stylet à la position voulue, on serre la vis et on laisse le stylet reprendre sa position de repos, après quoi, en manipulant le curseur droit on procède anx mensurations. Pour chaque changement de point initial ou de direction, on procède de la même manière.

Notre prototype a été executé en bois et d'une manière assez rustique, néanmoins il donne de bons résultats, assure une précision au $1 / 2 \mathrm{~mm}$ et sans qu'on ait encore acquis l'habitude de le manier, réduit le temps des mensurations de plusieures fois.

Comme nous l'avons dit e'est un prototype; s'il est adopté par les chercheurs il y aura naturellement de nombreux perfectionnements à $\mathrm{y}$ apporter et nous serions très heureux de recevoir des critiques et des suggestions dans ce sens.

Quelques jours avant la mise sous presse de cette communication nous avons obtenu un prototype exécuté en acier inoxydable, dont nous présentons la photographie (photo 2).

\section{SUMÁRIO}

A obtenção de medidas biométricas, em peixes, tem preocupado inúmeros pesquisadores. Apesar da existência de diversos aparelhos engrenhosos a tal fim destinados, observa-se que a obtenção dessas medidas constitue ainda questão das mais delicadas, sobretudo porque a maioria dos instrumentos até agora aparecidos, além de não assegurarem perfeita exatidão, distinguem-se, geralmente, pela sua extrema complexidade e custo sobremodo elevado. Baseado em tais cireunstâncias, vem o autor procurando obter um aparelho prático, destinado à medição de peixes, tendo recentemente idealizado uma régua que se lhe afigura atender às exigências em causa e cujo simples exame está a dispensar maiores deserięões. No entretanto, como a experiência demonstrou ao autor que para ehegar aos resultados finais foi necessário superar não pequenas dificuldades, deliberou deserever o protótipo, antes mesmo da construção do modêlo definitivo, em metal. Dessa maneira, acredita o autor poder assim limitar desperdícios de tempo da parte de pessoas interessadas nesse aparelho e de quem, com prazer, receberá críticas e sugestões tendentes a aperfeiçoá-lo. 


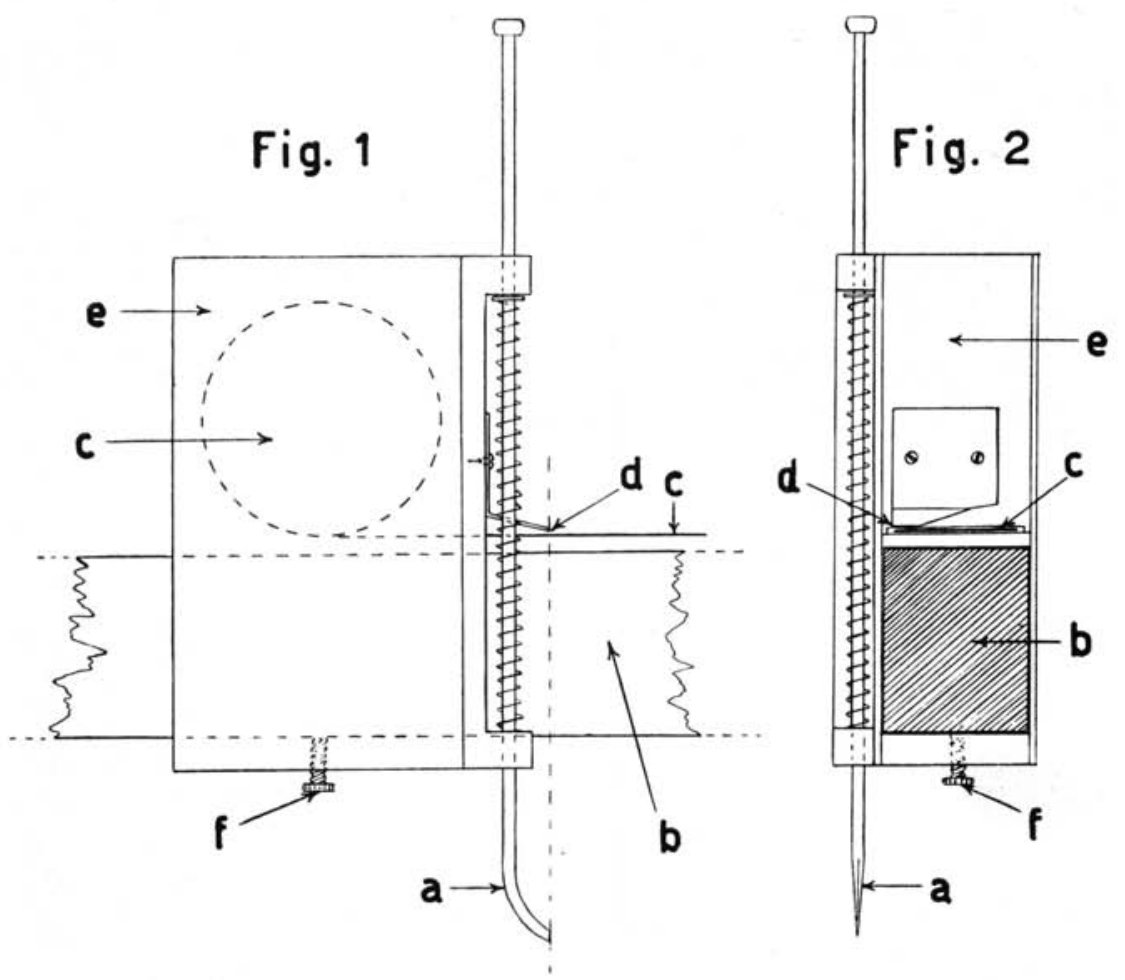




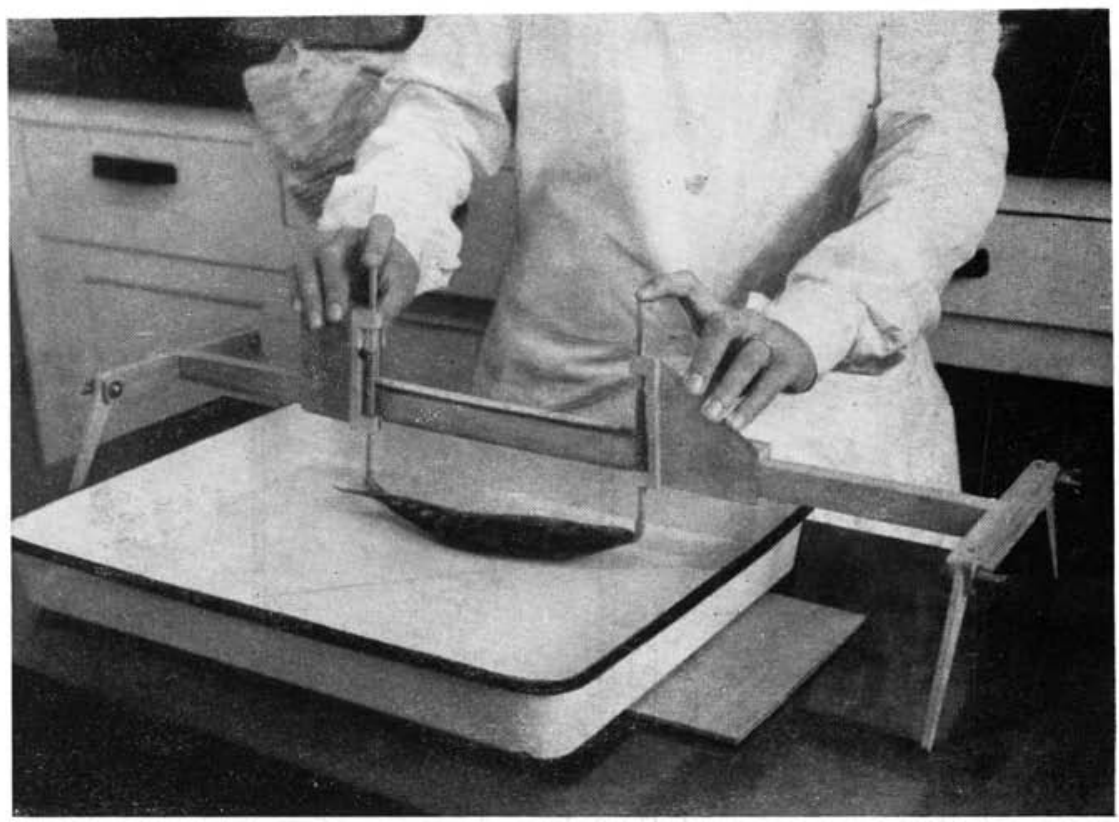

Photo. 1 - Prototype du dispositif, construit en bois.

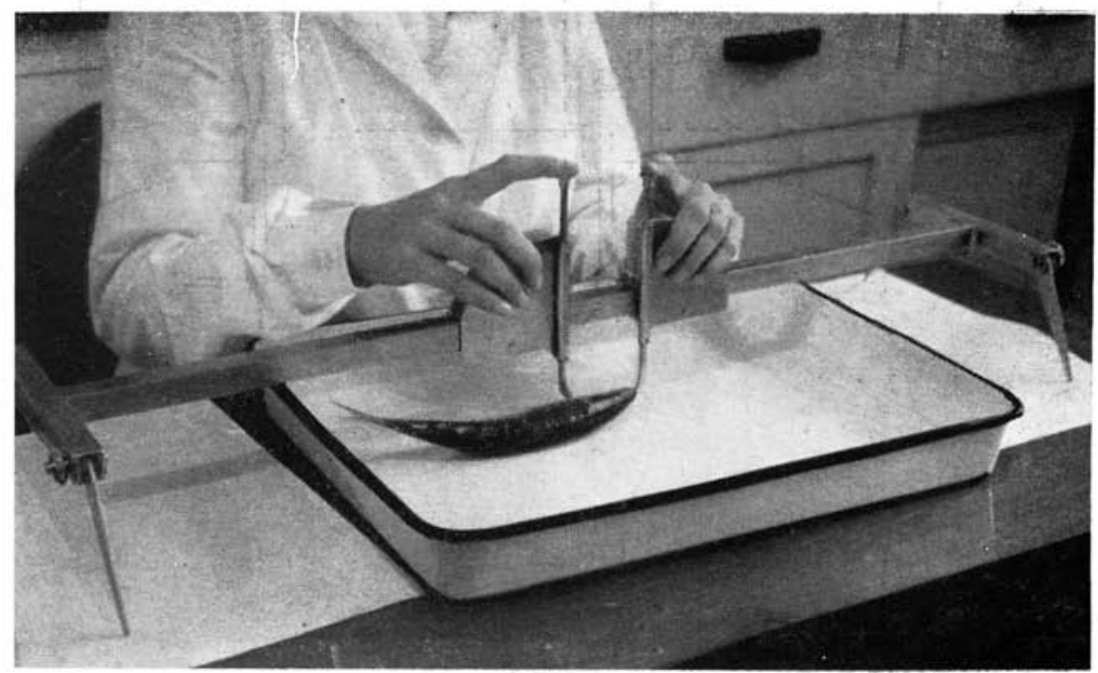

Photo. 2 - Travaux biométriques realisés avec le nouveau dispositif pour mensuration. 